\title{
A REFLECTION PRINCIPLE FOR SOLUTIONS TO THE HELMHOLTZ EQUATION AND AN APPLICATION TO THE INVERSE SCATTERING PROBLEM $\dagger$
}

\author{
by DAVID COLTON
}

(Received 26 November, 1975; revised 21 February 1976)

1. Introduction. A classical result in potential theory is the Schwarz reflection principle for solutions of Laplace's equation which vanish on a portion of a spherical boundary. The question naturally arises whether or not such a property is also true for solutions of the Helmholtz equation. This has been answered in the affirmative by Diaz and Ludford ([4]; see also [10]) in the limiting case of the plane. It is the purpose of this paper to show that a reflection principle is also valid for spheres of finite radius. As an application of this result we shall study the problem of the analytic continuation of solutions to the Helmholtz equation defined in the exterior of a bounded domain in three-dimensional Euclidean space $\mathbb{R}^{3}$. We shall show that through the use of the reflection principle derived in this paper, this problem can be reduced to the problem of the analytic continuation of an analytic function of two complex variables, which in turn can be performed through a variety of known methods (cf. [7]).

2. Integral operators and the reflection principle. We consider solutions of the Helmholtz equation

$$
\Delta_{n} u+\lambda u=0
$$

defined in $D \backslash \bar{S}$ where $D$ is a bounded starlike domain containing the open ball

$$
S=\left\{\mathbf{x}: r=\sqrt{x_{1}^{2}+\ldots+x_{n}^{2}}<a\right\}, \quad \mathrm{x}=\left(x_{1}, \ldots, x_{n}\right) .
$$

On the surface $r=a$ we assume that $u(r, \theta)=u(\mathbf{x})$ continuously assumes the boundary data

$$
u(a, \theta)=0,
$$

where $(r, \theta)=\left(r, \theta_{1}, \ldots, \theta_{n-1}\right)$ are spherical coordinates.

We shall obtain a reflection principle for solutions of (2.1), (2.2) through the use of an integral operator which maps harmonic functions defined in $D \mid \bar{S}$ and vanishing on $r=a$ onto solutions of (2.1), (2.2). In this connection our approach resembles in some way the " method of ascent " as developed by Gilbert [8], Eichler [5] and Colton and Wendland [3], except that we are now concerned with solutions defined in a multiply connected domain instead of a simply connected domain.

We look for a solution of (2.1) in the form

$$
u(r, \theta)=h(r, \theta)+\int_{a}^{r} s^{n-3} K(r, s ; \lambda) h(s, \theta) d s,
$$

† This research was supported in part by AFOSR Grant 76-2879.

Glasgow Math. J. 18 (1977) 125-130. 
where $h(r, \theta) \in C^{2}(D \backslash \bar{S}) \cap C^{0}(D \backslash S)$ is a solution of

$$
\Delta_{n} h=0
$$

such that

$$
h(a, \theta)=0 \text {. }
$$

Substituting (2.3) into (2.1) and integrating by parts using (2.5) we can show that (2.3) will be a solution of (2.1) provided $K(r, s ; \lambda)$ satisfies

$$
r^{2}\left[K_{r r}+\frac{(n-1)}{r} K_{r}+\lambda K\right]=s^{2}\left[K_{s s}+\frac{(n-1)}{s} K_{s}\right]
$$

and the initial data

$$
\begin{aligned}
& K(r, r ; \lambda)=-\frac{\lambda}{4} r^{2-n}\left(r^{2}-a^{2}\right) \\
& K(r, a ; \lambda)=0
\end{aligned}
$$

Setting

$$
\begin{aligned}
& \xi=\log r \\
& \eta=\log s
\end{aligned}
$$

we transform (2.6)-(2.8) into the initial value problem

$$
\begin{gathered}
M_{\xi \xi}-M_{\eta \eta}+\lambda e^{2 \xi} M=0 \\
M(\xi, \log a ; \lambda)=0 \\
M(\xi, \xi ; \lambda)=-\frac{\lambda}{4}\left(e^{2 \xi}-a^{2}\right)
\end{gathered}
$$

for the function

$$
M(\xi, \eta ; \lambda)=\exp \left\{\frac{(n-2)}{2}(\xi+\eta)\right\} K\left(e^{\xi}, e^{\eta} ; \lambda\right)
$$

defined in the cone $\{(\xi, \eta): \xi \leqq \eta, \eta \leqq \log a$, or $\xi \geqq \eta, \eta \geqq \log a\}$. (2.10)-(2.12) is a Goursat problem for a hyperbolic equation and has a unique (analytic) solution in this cone (cf. [6, pp. 118-119]). Hence we can conclude that the operator (2.3) exists. It is easy to show (cf. [2]) that if $u(r, \theta) \in C^{2}(D \backslash \bar{S}) \cap C^{0}(D \backslash S)$ is any solution of $(2.1),(2.2)$, then $u(r, \theta)$ can be represented in the form (2.3) for some harmonic function satisfying (2.5).

Before turning to the proof of the reflection principle for solutions $u(r, \theta)$ satisfying $(2.1)$, (2.2), we take this opportunity to construct another integral operator which in a sense is complementary to (2.3) and which we shall use in the next section of this paper. This operator is of the form

$$
u(r, \theta)=h(r, \theta)+\int_{a}^{r} s^{n-3} \tilde{K}(r, s ; \lambda) h(s, \theta) d s
$$


where $h(r, \theta) \in C^{2}(D \backslash \bar{S}) \cap C^{1}(D \backslash S)$ is a solution of (2.4) such that

$$
h_{r}(a, \theta)+\frac{(n-2)}{2 a} h(a, \theta)=0 .
$$

In order for $u(r, \theta)$ as defined by (2.14) to be a solution of $(2.1)$ we must have that $\tilde{K}(r, s ; \lambda)$ is a solution of (2.6) satisfying (2.7) and the initial data

$$
\widetilde{K}_{s}(r, a ; \lambda)+\frac{(n-2)}{2 a} \tilde{K}(r, a ; \lambda)=0 .
$$

This can be verified directly by substituting (2.14) into (2.1) and integrating by parts using (2.15). Using the change of variables (2.9) and setting

$$
\tilde{M}(\xi, \eta ; \lambda)=\exp \left\{\frac{(n-2)}{2}(\xi+\eta)\right\} \tilde{K}\left(e^{\xi}, e^{\eta} ; \lambda\right)
$$

we obtain the initial value problem

$$
\begin{gathered}
\tilde{M}_{\xi \xi}-\tilde{M}_{\eta \eta}+\lambda e^{2 \xi} \tilde{M}=0 \\
\tilde{M}_{\eta}(\xi, \log a ; \lambda)=0 \\
\tilde{M}(\xi, \xi ; \lambda)=-\frac{\lambda}{4}\left(e^{2 \xi}-a^{2}\right) .
\end{gathered}
$$

To solve (2.18)-(2.20) we introduce the function $E(\xi, \eta ; \lambda)$ defined as the (unique) solution of the characteristic initial value problem

$$
\begin{gathered}
E_{\xi \xi}-E_{\eta \eta}+\lambda e^{2 \xi} E=0 \\
E(\xi, \xi ; \lambda)=-\frac{\lambda}{4}\left(e^{2 \xi}-a^{2}\right) \\
E(\xi,-\xi+2 \log a ; \lambda)=-\frac{\lambda}{4}\left(e^{2 \xi}-a^{2}\right) .
\end{gathered}
$$

The existence of a unique (analytic) solution to (2.21)-(2.23) in the cone $\{(\xi, \eta): \xi \leqq \eta$, $\eta+\xi \leqq 2 \log a$, or $\xi \geqq \eta, \eta+\xi \geqq 2 \log a\}$ follows from standard results on hyperbolic equations (cf. [6, pp. 118-119]). A solution of (2.18)-(2.20) is now given by

$$
\bar{M}(\xi, \eta ; \lambda)=\frac{1}{2}[E(\xi, \eta ; \lambda)+E(\xi,-\eta+2 \log a ; \lambda)]
$$

and we have established the existence of the operator (2.14). It is again easy to show that if $u(r, \theta) \in C^{2}(D \backslash \bar{S}) \cap C^{1}(D \backslash S)$ is any solution of (2.1) satisfying

$$
u_{r}(a, \theta)+\frac{(n-2)}{2 a} u(a, \theta)=0
$$

then $u(r, \theta)$ can be represented in the form (2.14) for some harmonic function satisfying (2.15). 
We are now in a position to prove the following reflection principle for solutions of (2.1), (2.2):

TheOREM 1. Let $u(r, \theta) \in C^{2}(D \mid \bar{S}) \cap C^{0}(D \mid S)$ be a solution of $(2.1),(2.2)$ and let $D^{*}$ denote the set obtained by inverting $D \backslash S$ across $\partial S$; i.e. $(r, \theta) \in D^{*}$ if and only if $\left(\frac{a^{2}}{r}, \theta\right) \in D \backslash S$. Then $u(r, \theta)$ is a twice continuously differentiable (and hence analytic) solution of (2.1) in $D \mid S \cup D^{*}$.

REMaRK. The fact that twice continuously differentiable solutions of (2.1) are in fact analytic follows from classical regularity theorems for solutions of (2.1) (cf. [6]).

Proof of Theorem. From our previous discussion we can represent $u(r, \theta)$ in the form (2.3) where $h(r, \theta)$ satisfies $(2.4)$ and (2.5). Furthermore $u(r, \theta) \in C^{2}(D \backslash S) \cap C^{\circ}(D \backslash S)$ implies that $h(r, \theta) \in C^{2}(D \mid \bar{S}) \cap C^{0}(D \mid S)$. Hence from the reflection principle for harmonic functions $h(r, \theta)$ is harmonic in $D \mid S \cup D^{*}$, and so by $(2.3) u(r, \theta)$ is twice continuously differentiable in $D \mid S \cup D^{*}$.

\section{An application to the Inverse Scattering Problem.}

Suppose an incoming plane acoustic wave of frequency $\omega$ moving in the direction of the $z$-axis is scattered off a " soft " bounded obstacle $\Omega$ in $\mathbb{R}^{3}$ and that $u(r, \theta, \phi) e^{i \omega t}$ is the velocity potential of the scattered wave, where $(r, \theta, \phi)$ denote spherical coordinates. Then $u(r, \theta, \phi)$ will be a solution of (2.1) in the exterior of $\Omega$ for $n=3, \lambda=\frac{\omega^{2}}{c^{2}}=k^{2}$, where $c$ is the speed of sound. At infinity $u(r, \theta, \phi)$ has the asymptotic behaviour

$$
u(r, \theta, \phi) \sim \frac{e^{i k r}}{r} f(\theta, \phi)
$$

where $f(\theta, \phi)$ is the far field pattern (cf. [13]). The inverse scattering problem is to determine $\Omega$, given the fact that $f(\theta, \phi)$ is known exactly. From the results of Müller [13] we can determine $u(r, \theta, \phi)$ outside the smallest ball $S$ containing $\Omega$ in its interior, where $S$ can be determined from a knowledge of $f(\theta, \phi)$. In particular if the radius of $S$ is $a$, one can write ([9], [13])

$$
u(r, \theta, \phi)=\sum_{n=0}^{\infty} \sum_{m=-n}^{n} a_{n m} h_{n}^{(1)}(k r) S_{n m}(\theta, \phi) \quad(r \geqq a)
$$

where the coefficients $a_{n m}$ are determined from the far field pattern $f(\theta, \phi), h_{n}^{(1)}$ denotes a spherical Hankel function, $S_{n m}$ a spherical harmonic, and the series (3.2) is uniformly convergent for $r \geqq a, 0 \leqq \theta \leqq \pi, 0 \leqq \phi \leqq 2 \pi$. Hence to find $\Omega$ we must analytically continue $u(r, \theta, \phi)$ as given by (3.2) across the boundary of $S$ and look for the locus of points where $u(r, \theta, \phi)+\exp (i k r \cos \theta)=0$. This problem of analytic continuation has been studied by many research workers, in particular, Weston, Bowman and Ar [15], Colton [1], Sleeman [14], Millar [12] and Hartman and Wilcox [9]. In this section we shall contribute to this study by using the integral operators and reflection principle of section 2 to relate the domain of regularity of $u(r, \theta, \phi)$ to that of an analytic function of two complex variables. The advantage of such a relationship is that once this has been done there is a number of known methods for 
determining the domain of regularity of analytic functions of several complex variables; in particular see [7, section 1.3]. We first prove the following theorem. (Compare this result to that of Millar in the simpler case of two dimensions ([11]).)

THEOREM 2. Let $h(r, \theta, \phi)$ be the (unique) harmonic function defined in the exterior of the ball $S$ such that $h(a, \theta, \phi)=u(a, \theta, \phi)$ on $\partial S$. If $h(r, \theta, \phi)$ can be continued to a harmonic function defined in the exterior of a starlike domain $D \subset S$, then $u(r, \theta, \phi)$ can be continued as a solution of (2.1) (with $n=3, \lambda=k^{2}$ ) into the exterior of $D$.

Proof. Let $\tilde{h}(r, \theta, \phi)$ be the harmonic function defined by

$$
\left.\tilde{h}(r, \theta, \phi)=\frac{1}{2}\left[h(r, \theta, \phi)+\left(\frac{a}{r}\right) h\left(\frac{a^{2}}{r}\right), \theta, \phi\right)\right],
$$

where we have made use of Kelvin's inversion formula. Then $\tilde{h}(r, \theta, \phi)$ is regular in $D^{*} \cup S \backslash D$ where $D^{*}$ denotes the set obtained by inverting $\bar{S} \mid D$ across $\partial S$. Furthermore we have

$$
\tilde{h}_{r}(a, \theta, \phi)+\frac{1}{2 a} \tilde{h}(a, \theta, \phi)=0 .
$$

Hence from (2.14) we have that

$$
\tilde{u}(r, \theta, \phi)=\tilde{h}(r, \theta, \phi)+\int_{a}^{r} \tilde{K}\left(r, s ; k^{2}\right) \tilde{h}(s, \theta, \phi) d s
$$

is a solution of

on $D^{*} \cup S \backslash D$ and

$$
\Delta_{3} u+k^{2} u=0
$$

$$
\tilde{u}(a, \theta, \phi)=u(a, \theta, \phi) .
$$

Therefore $w(r, \theta, \phi)=u(r, \theta, \phi)-\tilde{u}(r, \theta, \phi)$ is a solution of (3.6) in $D^{*}$ such that $w(a, \theta, \phi)=0$, and hence, by Theorem $1, w(r, \theta, \phi)$ is an analytic solution of (3.6) in $D^{*} \cup S \backslash D$. We can now conclude that $u(r, \theta, \phi)$ is analytic in $D^{*} \cup S \backslash D$ and since $u(r, \theta, \phi)$ is already known to be analytic in the exterior of $S$, the theorem follows.

In order to apply Theorem 2 it is necessary to have a method for determining the location of the singularities of the harmonic function $h(r, \theta, \phi)$. However, this theory has been extensively developed by Gilbert in [7] and [8]. In particular since

$$
h(r, \theta, \phi)=\sum_{n=0}^{\infty} \sum_{m=-n}^{n} a_{n m} h_{n}^{(1)}(k a)\left(\frac{r}{a}\right)^{-n-1} S_{n m}(\theta, \phi)
$$

for $r \geqq a, 0 \leqq \theta \leqq \pi, 0 \leqq \phi \leqq 2 \pi$, we have (cf. [7, chapter 3], or [8, chapter 7]) that the singular points of $h(r, \theta, \phi)$ can be determined from a knowledge of the singular points of the analytic function of two complex variables

$$
g\left(z_{1}, z_{2}\right)=\sum_{n=0}^{\infty} \sum_{m=-n}^{n} a_{n m} h_{n}^{(1)}(k a) z_{1}^{n} z_{2}^{m}
$$

As previously pointed out, methods for determining the singular points of (3.9) can be found in 
[7]. It should be observed that in the case when $u(r, \theta, \phi)=u(r, \theta)$ is axially symmetric (i.e. independent of $\phi)$ then $g\left(z_{1}, z_{2}\right)=g\left(z_{1}\right)$ is an analytic function of a single complex variable, and all calculations are considerably simplified (cf. [1]).

\section{REFERENCES}

1. D. Colton, On the inverse scattering problem for axially symmetric solutions of the Helmholtz equation, Quart. J. Math. (Ser. 2) 22 (1971), 125-130.

2. D. Colton, Integral operators and reflection principles for parabolic equations in one space variable, J. Differential Equations 15 (1974), 551-559.

3. D. Colton and W. Wendland, Constructive methods for solving the exterior Neumann problem for the reduced wave equation in a spherically symmetric medium, Proc. Roy. Soc. Edinburgh Sect. $A$, 75 (1976), 97-107.

4. J. B. Diaz and G. S. S. Ludford, Reflection principles for linear elliptic second order partial differential equations with constant coefficients, Ann. Mat. Pura. Appl. 39 (1955), 87-95.

5. M. Eichler, On the differential equations $u_{x x}+u_{y y}+N(x) u=0$, Trans. Amer. Math. Soc. 65 (1949), 259-278.

6. P. R. Garabedian, Partial differential equations (John Wiley, 1964).

7. R. P. Gilbert, Function theoretic methods in partial differential equations (Academic Press, 1969).

8. R. P. Gilbert, Constructive methods for elliptic equations. Lecture Notes in Mathematics, No. 365 (Springer-Verlag, 1974).

9. P. Hartman and C. Wilcox, On solutions of the Helmholtz equation in exterior domains, Math. Z. 75 (1961), 228-255.

10. F. John, Continuation and reflection of solutions to partial differential equations, Bull. Amer. Math. Soc. 63 (1957), 327-344.

11. R. F. Millar, Singularities of two-dimensional exterior solutions of the Helmholtz equation, Proc. Camb. Phil. Soc. 69 (1971), 175-188.

12. R. F. Millar, Singularities of solutions to exterior analytic boundary value problems for the Helmholtz equation in three independent variables I. The plane boundary, SIAM. J. Math. Anal. 7(1976), 131-156.

13. C. Müller, Radiation patterns and radiation fields, J. Rat. Mech. Anal. 4 (1955), 235-246.

14. B. D. Sleeman, The three-dimensional inverse scattering problem for the Helmholtz equation, Proc. Camb. Phil. Soc. 73 (1973), 477-488.

15. V. H. Weston, J. J. Bowman and E. Ar, On the inverse electromagnetic scattering problem, Arch. Rat. Mech. Anal. 31 (1968), 199-213.

UNIVERSITY OF STRATHCLYDE

Glasgow, Scotland 